\title{
ON THE ZEROS OF INFRAPOLYNOMIALS FOR PARTLY ARBITRARY POINT SETS ${ }^{1}$
}

\author{
MORRIS MARDEN
}

1. Introduction. Let $S$ be a compact point set and let $p(z)=z^{n}$ $+a_{1} z^{n-1}+\cdots+a_{n}$ be an infrapolynomial on $S$ in the sense that it has no underpolynomial on $S$. (A polynomial $q(z)=z^{n}+b_{1} z^{n-1}+\cdots$ $+b_{n}$ is said to be an underpolynomial of $p(z)$ on $S$ if $q(z)=0$ on the subset $S^{\prime}$ of $S$ where $p(z)=0$ and if $|q(z)|<|p(z)|$ on $S-S^{\prime}$.) Fejér $[1]^{2}$ has proved that all the zeros of $p(z)$ lie in the convex hull $K(S)$ of $S$. His theorem is analogous to the theorem of Lucas ${ }^{3}$ that the zeros of the derivative of a polynomial $f(z)$ lie in the convex hull of the set of the zeros of $f(z)$.

Various other theorems have been proved concerning the location of the zeros of an infrapolynomial. Like Fejer's theorem, many are analogous to known theorems on the location of all the zeros of the derivative of a polynomial $f(z)$ when the positions of all the zeros of $f(z)$ are prescribed.

However, in the literature there are a number of theorems concerning the location of some of the zeros of the derivative of a polynomial $f(z)$ when the positions of only some of the zeros of $f(z)$ are prescribed. An example is the theorem of Grace and Heawood ${ }^{4}$ that, if $z_{1}$ and $z_{2}$ are any two distinct zeros of an $n$th degree polynomial $f(z)$, at least one zero of the derivative of $f(z)$ lies in the circle with center at $(1 / 2)\left(z_{1}+z_{2}\right)$ and radius $(1 / 2)\left|z_{1}-z_{2}\right| \cot (\pi / n)$. Another example is the theorem due to Marden ${ }^{5}$ that, if $K$ zeros $(2 \leqq K \leqq n)$ of an $n$th degree polynomial $f(z)$ lie in a circle of radius $R$, at least $K-1$ zeros of $f^{\prime}(z)$ lie in the concentric circle of radius $R \csc [\pi / 2(n-K+1)]$.

It is the object of the present note to develop for infrapolynomials results analogous to these theorems of Grace-Heawood and Marden. The results involve the location of some of the zeros of an infrapoly-

Presented to the Society, August 29, 1958; received by the editors July 21, 1958 and, in revised form, October 8, 1958.

1 The research reported here was sponsored by the United States Army under Contract No. DA-11-022-ORD-2059 and conducted at the U. S. Army Mathematics Center, University of Wisconsin, Madison, Wisconsin.

${ }^{2}$ A number enclosed in a square bracket refers to the bibliography at the close of this note.

${ }^{3}$ See $[4$, pp. 14-16].

See [4, pp. 84-85].

6 See $[3$, p. 364$]$ or $[4$, p. 90$]$. 
nomial for a set $S$ comprised of points, only some of which have prescribed positions.

2. ThEOREM 1. Let $p(z)=z^{n}+a_{1} z^{n-1}+\cdots+a_{n}$ be an infrapolynomial on the set $S=S_{0}+S_{1}$ where $S_{0}$ is a compact point set (finite or infinite) and $S_{1}$ is a set of $k$ points, $0 \leqq k \leqq n$. Let $T_{0}$ be the set comprised of all points from which $S_{0}$ subtends an angle of at least $\pi /(k+1)$. If $p(z) \neq 0$ on $S$, then $p(z)$ has at most $k$ zeros outside $T_{0}$ irrespective of the location of $S_{1}$.

Proof. We shall make use of the following result due to Fekete [2].

Fenete's Theorem. Let $p(z)=z^{n}+a_{1} z^{n-1}+\cdots+a_{n}$ be an infrapolynomial on $S$. If $p(z) \neq 0$ on $S$, then there exist $(m+1)$ positive constants $\lambda_{j}$ with $\lambda_{0}+\lambda_{1}+\cdots+\lambda_{m}=1$ and $(m+1)$ points $z_{j}$ in $S$ such that $p(z)$ is a factor of the polynomial

$$
\Omega(z)=\omega(z) \sum_{j=0}^{m}\left[\lambda_{j} /\left(z-z_{j}\right)\right], \quad n \leqq m \leqq 2 n,
$$

where

$$
\omega(z)=\left(z-z_{0}\right)\left(z-z_{1}\right) \cdots\left(z-z_{m}\right) .
$$

It follows that the zeros of $p(z)$ satisfy an equation of the form

$$
\sum_{j=0}^{m} \lambda_{j} /\left(z-z_{j}\right)=0 .
$$

Let us suppose that $p(z)$ has $k+1$ zeros, $Z_{0}, Z_{1}, \cdots, Z_{k}$ outside $T_{0}$. Let $(2 h)$ be the shortest distance of any of the points $Z_{j}$ to the boundary of $T_{0}$. The zeros of (2.1) are continuous functions of the $\lambda_{j}$. If we choose $\epsilon$ as a sufficiently small number, $0<\epsilon<h$, we can find a $\delta>0$ such that for rational numbers $\rho_{j}$ with $\left|\rho_{j}-\lambda_{j}\right|<\delta, j=0,1$, $\cdots, m$, the equation

$$
\sum_{j=0}^{m}\left[\rho_{j} /\left(z-z_{j}\right)\right]=0
$$

has roots $\zeta_{0}, \zeta_{1}, \cdots, \zeta_{k}$ with $\left|\zeta_{j}-Z_{j}\right|<\epsilon$ for $j=0,1, \cdots, m$. Thus the points $\zeta_{0}, \zeta_{1}, \cdots, \zeta_{k}$ also lie outside $T_{0}$.

Let us next choose a positive, sufficiently large integer $N$ so that each of the quantities $\nu_{j}=\rho_{j} N$ is an integer. The equation

$$
\sum_{j=0}^{m}\left[\nu_{j} /\left(z-z_{j}\right)\right]=0
$$

which has also the roots $\zeta_{0}, \zeta_{1}, \cdots, \zeta_{k}$, is satisfied by those zeros of 
the derivative of the polynomial $f(z)=\prod_{j=0}^{m}\left(z-z_{j}\right)^{\nu_{j}}$ which are distinct from the $z_{j}$.

Since all that is known about the $z_{j}$ is that each lies in $S$, some of them may be points of $S_{1}$. But the set $S_{1}$ contains only $k$ points so that at least $m+1-k$ of the $z_{j}$ do not lie in $S_{1}$. Let us, if necessary, relabel the $z_{j}$ so that the points $z_{0}, z_{1}, \cdots, z_{m-k}$ all lie in $S_{0}$. Now any set of $m+2$ distinct points comprised of $m-k+1$ zeros $z_{0}$, $z_{1}, \cdots, z_{m-k}$ of $f(z)$ and $k+1$ zeros $\zeta_{0}, \zeta_{1}, \cdots, \zeta_{k}$ of $f^{\prime}(z)$, are known ${ }^{6}$ to satisfy an identity of the form

$$
\sum M_{j_{0} j_{1}} \cdots j_{k}\left[\left(\zeta_{0}-z_{j_{0}}\right)\left(\zeta_{1}-z_{j_{1}}\right) \cdots\left(\zeta_{k}-z_{j_{k}}\right)\right]^{-1}=0
$$

where the $j_{0}, j_{1}, \cdots, j_{k}$ run independently through the values $0,1, \cdots, m-k$ and the numbers $M_{j_{0} j_{1}} \cdots j_{k}$ are all positive.

Since $\zeta_{0}, \zeta_{1}, \zeta_{k}$ all lie outside $T_{0}$, points $\tau_{0}, \tau_{1}, \cdots, \tau_{k}$ may be found in $S_{0}$ so that for each $i$

$$
0<\arg \left[\left(\zeta_{i}-\tau_{i}\right) /\left(\zeta_{i}-z_{j_{i}}\right)\right]<\pi /(k+1) .
$$

If the left-side of (2.4) is multiplied by $\left(\zeta_{0}-\tau_{0}\right)\left(\zeta_{1}-\tau_{1}\right) \cdots\left(\zeta_{k}-\tau_{k}\right)$, each term in the resulting sum has, due to $(2.5)$, the property

$$
0<\arg \prod_{i=0}^{k}\left[\left(\zeta_{i}-\tau_{i}\right) /\left(\zeta_{i}-z_{j_{i}}\right)\right]<\pi .
$$

Hence the sum in (2.4) cannot vanish and so at most $k$ zeros of any infrapolynomial for $S$ can lie outside of $T_{0}$.

3. Corollaries. Fejér's Theorem, stated in $\$ 1$, is the special case of Theorem 1 corresponding to $k=0$. Other immediate corollaries are the following:

COROLlaRy 1. Let $S_{0}$ be a compact point set all of the points of which lie in a circle $C$ of radius $R$. Let $S$ be the point set comprised of $S_{0}$ and $k$ additional points, the latter having arbitrary positions in the complex plane. Then any infrapolynomial $p(z)=z^{n}+a_{1} z^{n-1}+\cdots+a_{n} \neq 0$ on $S$ has at least $n-k$ zeros within the concentric circle of radius $R \csc [\pi / 2(k+1)]$.

Corollary 2. Let $S_{0}$ be a compact set situated on the line segment joining the points $z=a$ and $z=b$ of the real axis. Let $S$ be comprised of $S_{0}$ and $k$ additional points situated at random in the plane. If $p(z)$ $=z^{n}+\cdots$ is any nonvanishing infrapolynomial on $S$, then at least $n-k$ zeros of $p(z)$ lie in region bounded by the two circles with radius $L \csc \pi /(k+1)$ and centers $z=c \pm i L \cot [\pi /(k+1)]$ where $c=(1 / 2)(a+b)$ and $L=(1 / 2)|a-b|$.

- See [3, pp. 356-364]. 
4. Generalization. Theorem 1 may be extended to the case that $p(z)$ has some zeros on $S$, as follows.

THEOREM 2. In the notation of Theorem 1 , if $p(z)$ has $\nu$ zeros on $S_{1}$ where $0 \leqq \nu \leqq \min (k, n-k)$, then $p(z)$ has at most $\nu+k$ zeros (counted with their multiplicities) outside $T_{0}$ irrespective of the location of $S_{1}$.

Proof. Let us assume that $p(z)$ has zeros both on $S_{0}$ and $S_{1}$ and denote these by $\xi_{1}, \xi_{2}, \cdots, \xi_{\mu}$ and $\zeta_{1}, \zeta_{2}, \cdots, \zeta_{\nu}$ respectively where $0 \leqq \mu \leqq n, 0 \leqq \nu \leqq n, \mu+\nu \leqq \min (k, n-k)$. Let us write

$$
p(z)=\left(z-\xi_{1}\right) \cdots\left(z-\xi_{\mu}\right)\left(z-\zeta_{1}\right) \cdots\left(z-\zeta_{\nu}\right) p_{1}(z)
$$

where $p_{1}(z) \neq 0$ on $S$. Then, relative to polynomials of degree $n-\mu-\nu$, $p_{1}(z)$ is an infrapolynomial on $S$, since otherwise $p_{1}(z)$ would have an underpolynomial $q_{1}(z)$ and thus $p(z)$ would have the underpolynomial $q(z)=\left[p(z) / p_{1}(z)\right] q_{1}(z)$.

On applying Theorem 1 , we learn that $p_{1}(z)$ has at least $n-\mu-\nu-k$ zeros on $T_{0}$. But, as $S_{0} \subset T_{0}, p(z)$ has as zeros in $T_{0}$ the points $\xi_{j}$ as well as the zeros of $p_{1}(z)$. Thus $p(z)$ has at least $n-\nu-k$ zeros in $T_{0}$, as was to be proved.

\section{BiBLIOGRAPHY}

1. L. Fejér, Uber die Lage der Nullstellen von Polynomen, die aus Minumumforderungen gewisser Art entspringen, Math. Ann. vol. 85 (1922) pp. 41-48.

2. M. Fekete, On the structure of extremal polynomials, Proc. Nat. Acad. Sci. U.S.A. vol. 31 (1951) pp. 95-103.

3. M. Marden, Kakeya's problem on the zeros of the derivative of a polynomial, Trans. Amer. Math. Soc. vol. 45 (1939) pp. 355-368.

4. - The geometry of the zeros of a polynomial in a complex variable, Mathematical Surveys No. 3, American Mathematical Society, 1949.

UNIVERSITY OF Wisconsin-MiLWAUkeE 